Pub. Mat. UAB

№ 17 , Febrer, 1980

\title{
POWERS OF THE AUGMENTATION IDEAL
}

Surmary of talk given at Universitat

Autònoma de Barcelona, 19 April, 1978, by B. Hartley.

Let $G$ be a group, $R$ a commutative ring with 1 (we will in fact only be concerned with the cases when $R$ is a field or $\mathbb{Z}$ ).

The group ring $R G$ is the set of normal finite linear combinations $\underset{g \varepsilon G}{\Sigma}{ }^{\lambda} g^{g}\left(\lambda_{g} \in R\right)$, with the definitions $\left(\Sigma \lambda_{g} g\right)+\left(\Sigma \mu_{g} g\right)=\Sigma\left(\lambda_{g}+\mu_{g}\right) g$ and $\left(\Sigma \lambda_{g} g\right)\left(\Sigma \nu_{h} h\right)=\sum_{k}\left(\underset{g h=k}{\sum} \lambda_{g}{ }_{h}\right) k$. The augmentation $\varepsilon: R G \rightarrow R$ given by $\varepsilon\left(\Sigma \lambda_{g} g\right)=\Sigma \lambda_{g}$ is a ring homomorphism and its kernel $\Delta(R, G)$ is the augmentation ideal of $R G$. We are interested in the powers $\Delta^{n}(R, G)$ and the associated dimension subgroups

$$
D_{n}(R, G)=G \cap\left(1+\Delta^{n}(R, G)\right)
$$

Note that $D_{n}(R, G)$ is the kernel of the natural map of $G$ into the group of units $U\left(R G / \Delta^{n}(R, G)\right)$ of $R G / \Delta^{n}(R, G)$.

This is not intended to be a detailed survey of this area, but simply a discussion of some sampie results.

\section{BACKGROUND}

First we mention some connections between this subject and other problems.

\section{Connection with matrix representations}

It is often useful to know that certain groups can be represented 
faithfully by matrices over some well behaved ring.

Theorem. If $G$ is a finiteiy generated $n i l p o t e n t$ group, then $G$ can be embedded in some $\mathrm{GL}(n, \mathbb{Z})$ (much more general results are true).

Indication of proof. We easily reduce to the case when $G$ is torsionfree. Let $G$ have nilpotency class $c$. Then $D_{C+1}(\mathbb{Z}, G)=1$ (quite a difficult result). Hence $G$ can be embedded in the group of units of $\mathbb{Z} G / \Delta^{C+1}(\mathbb{Z}, G)=A$. The additive group of $A$ is finitely generated, and $G$ operates faithfully on this additive group by right multiplication. The additive torsion subgroup $T$ of $A$ is finite, and it is easy to see that $G$ operates faithfully on $A / T$.

See: P. Hall "The Edmonton Notes on Nilpotent Groups" (Queen Mary College, London) for the details.

2. Connection with residual nilpotency of groups

Theorem (Baumsiag, Passi, see J. Pure App. Algebra 6 (1975)). Let $F$ be a non-abelian free group, $R \triangleleft F$. Then $F / R^{\prime}$ is residually nilpotent if and only if $\prod_{n=1}^{\infty} \Delta(\mathbb{Z}, G)=0$, where $G=F / R$.

\section{DIMENSION SUBGROUPS}

For any $G$, let $G_{1}=G$ and $G_{n+1}=\left[G_{n}, G\right]$, so that $G_{1} \geq G_{2} \geq \ldots$ is the lower central series of $G$. Then

$$
\text { Lemrna 2.1. } G_{n} \leq D_{n}(R, G)
$$

This follows. by induction from the identity

$$
1 \sim x^{-1} y^{-1} x y=x^{-1} y^{-1}((y-1)(x-1)-(x-1)(y-1)) .
$$


Let $\sqrt{G_{n}} / G_{n}$ be the torsion subgroup of $G / G_{n}$.

Theorem 2.2. If $K$ is a field of characteristic zero, then $D_{n}(K, G)=$ $=\sqrt{G_{n}}$.

This is due to Jennings; a proof can be found in P. Hall's Edmonton Notes, or D.S. Passman "The Algebraic Structure of Group Rings" (Interscience).

Theorem 2.3. (Lazard) If $K$ is a field of characteristic $p>0$, then

$$
D_{n}(K, G)=\underbrace{I I}_{i p^{j} \geq n} G_{i}^{p^{j}}
$$

where if $H$ is a group, $H^{m}=\left\langle h^{m}: h \in H\right\rangle$. Another description of $D_{n}(K, G)$ was given earlier by Jennings. Theorem 2.3 can also be found in Passman's book. Note that in Theorem 2.3, if $G$ has exponent $p$, then $D_{n}(K, G)=G_{n}$ for all $n$.

Theorem 2.2 and 2.3 show that over fields, the dimension subgroups can be completely described in group theoretic terms.

For the case $R=\mathbb{Z}$ the situation is more complicated.

-It was at one time conjectured that $D_{n}(Z, G)=G_{n}$ for all $G$ (Dimension subgroup conjecture) and several false proofs have been given.

Theorem 2.4. (i) If $F$ is free, then $D_{n}(F)=F_{n}$ for all $n$. (Magnus, 1937)

(ii) If $G$ is any group, then $D_{n}(G)=G_{n}$ for $n=1,2,3$ (I don't know who first proved these results; quite a number of proofs are now available, for example A.H.M. Hoare, J. London Math. Soc.) 
iii) If $G$ is a finite $p-$ group, then $D_{n}(G)=G_{n}$ for $n \leq p$ (Moran; Proc. Cambridge Philos. Soc.)

I have written $D_{n}(G)$ for $D_{n}(\mathbb{Z}, G)$.

Eventually the Dimension Subgroup Conjecture was refuted by

Theorem 2.5 (Rips, 1972) There exists a finite 2-group $G$ of class 3 such that $\left|D_{4}(G)\right|=2$. (Israel J. Math.)

The best result to date is contained in a recent and difficult paper of Sjogren (J. Pure Appd. Algebra (1979)).

Theorem 2.6 (Sjogren) There exists an (explicitly given) function $c_{n}$ such that if $G$ is any group, then $D_{n}(G) / G_{n}$ has exponent dividing $c_{n}$.

The proof uses some elementary spectrai sequence ideas and some complicated Lie-theoretic methods. I have a simplified version of the proof. We have $c_{1}=c_{2}=c_{3}=1, c_{4}=2$, so $2.4(i i)$ follows from Sjogren's work, and Rips' example shows that $c_{4}$ is best possible. Also by examining the function $c_{n}$ one can replace the restriction $n \leq p$ in 2.4 (iii) by $n \leq p+1$.

\section{Some open problems}

Problem 1. Does there exist an integer valued function $f(c)$ such that if $G$ is a nilpotent group of class.c, then $D_{f(c)}(G)=1$ ? Rips' example shows that $f(c)=c+1$ will not do, but there is no counterexample known to rule out the possibility $f(c)=c+2$.

Problem 2. (a weaker version of Problem 1). If $G$ is nilpotent, is it true that $\stackrel{\infty}{n=1}_{n} D_{n}(G)=1$ ? 
Problem 3 . Does there exist a finite p-group $(p \neq 2)$ such that $D_{n}(G) \neq$ $\neq G_{n}$ for some $n$ ? The smallest possibility is $p=3, n=5$.

III. THE. LIE RING AND THE GRADED RING

Let $G$ be a group, with lower central series

$$
G_{1} \geq G_{2} \geq \cdots
$$

We write $G_{n} / G_{n+1}$ additively and form the abelian group

$$
s(G)=\bigoplus_{n=1}^{\infty} G_{n} / G_{n+1}
$$

(restricted direct sum), and define an operation (, ) on $L(G)$ by

$$
\left(x G_{n+1}, y G_{m+1}\right)=[x, y] G_{n+m+1}
$$

( $\left.x \notin G_{n}, y \in G_{m}\right)$ and extending by additivity. This turns $\bar{L}(G)$ into a Lie ring; the Jacobi identity follows from the Witt identity.

Now writing $\Delta=\Delta(\mathbb{Z}, G)$, we form the abelian group

$$
G(G)=\bigoplus_{n=0}^{\infty} \Delta^{n} / \Delta^{n+1} \text {, }
$$

and define

$$
\left(\alpha+\Delta^{n+1}\right)\left(\beta+\Delta^{m+1}\right)=\alpha \beta+\Delta^{n+m+1} .
$$

We extend this operation by additivity again, and obtain this time an associative ring, called the associated graded ring of $\mathbb{Z} G$. We can think of $G(G)$ as a lie ring under usual uv-vu operation, and then the map

$$
\sigma: x G_{n+1} \rightarrow(x-1)+\Delta^{n+1} \quad\left\langle x \in G_{n}\right\}
$$


extends to a $L$ ie homomorphism of $L(G)$ into $G(G)$. The kernel of the restriction of $\phi$ to $G_{n} / G_{n+1}$ is $\left(D_{n+1}(G) \cap G_{n}\right) / G_{n+1}$, and so in this way we obtain a connection between dimension subgroups and Lie alnebras.

The map $\phi$ also determines a homomorphism from $\left.L(G) \mathbb{Z}^{K} \rightarrow Q G\right) \mathbb{Z}^{K}$, if $K$ is any field of characteristic zero, and we have the following beautiful result of Quillen (J. Algebra $10(1968)$ ).

Theorem 3.1. With the above notation, is injective and $G(G) \mathrm{K}$ is the universal enveloping algebra of $L(G) \otimes K$.

It is in fact more natural for these purposes to construct the Lie ring from the characteristic zero dimension series

$$
\sqrt{G_{1}} \geq \sqrt{G}_{2} \quad \ldots
$$

though the two Lie rings become isomorphicron tensoring with a field of characteristic zero. However using the dimension series gives the right. analogy with characteristic $p>0$; here we get a result like Theorem 3.1 involving the restricted universal enveloping algebra. For a more detailed survey of these matters, see I.B.S. Passi (J. London Math. Soc. 1979).

\section{POWERS OF THE AUGMENTATION IDEAL.}

Here we confine ourselves to the question: When is it true that ${ }_{n}^{\infty} \Delta^{n}=0$, where $\Delta=\Delta(\not \mathbb{Z}, G)$ ? In dealing with this question, it seems $n=1$ unavoidable to consider also the ring $\mathbb{Z} / \mathrm{p}^{\mathrm{m}} \mathbb{Z}$.

Theorem 4.1. $\sum_{n=1}^{\infty} \Delta^{n}\left(G, \mathbb{Z} / p^{m} \mathbb{Z}\right)=0$ if and only if $G$ is residually a nilpotent p-group of finite exponent. 
This was proved by B. Hartley (Proc. London Math. Soc. 1969) and also by K.И. Gruenberg (unpublished). The case $m=1$ was done much earlier by Mal'cev.

Theorem 4.2. If $G$ is residually torsion-free nilpotent, then $\bigcap_{n=1}^{\infty} \Delta^{n}(\mathbb{Z}, G)=0$.

This is also due to Hartley in the above paper. The ma in part of the proof consists of adapting a construction of $P$. Hall to obtain, for a finitely generated torsion-free nilpotent group $G$, a $\mathbb{Z}$-basis of $\mathbb{Z} G$ which is closely related to the powers of $\Delta$. This can now be done rather better. Let $\sqrt{\Delta^{n}}(\mathbb{Z}, G) / \Delta^{n}(\mathbb{Z}, G)$ denote the additive torsion subgroup of $\mathbb{Z} G / \Delta^{n}(\mathbb{Z}, G)$.

Theorem 4.3. Let $\mathrm{G}$ be a finitely generated torsion-free nilpotent group. Then $\mathbb{Z} G$ has a $\mathbb{Z}$-basis $B$ such that, for each $n \geq 1, \sqrt{A^{n}}(\mathbb{Z}, G)$ is spanned by a subset of $B$. (See Symposia Math., 1976)

other proofs of 4.2 have since been given by A.L. Smej ${ }^{*} k$ in using techniques from Lie Aigebras (Trans. Moscow Math. Soc. 1973, and also in a recent issue of Uspekhi Mat. Nauk.)

Finally, A.I. Lichtman showed that sufficient conditions given by 4.1 and 4.2 are very neariy necessary. If $G$ is a group, then we say that $G$ is discriminated by nilpotent p-groups of finite exponent if and only if for each finite set of elements $x_{1}, \ldots, x_{m} \varepsilon G$, there exists a prime $\rho$ and $a$ homomorphism $\theta$ of $G$ into a nilpotent $p$-group of finite exponent, such that

$$
\theta\left(x_{i}\right) \neq 1 \quad(1 \leq i \leq m)
$$


Theorem (Israel J. Math. 1977)

- $n \Delta^{n}(\mathbb{Z}, G)=0$ if and only if $G$ is either residually torsion$n=1$

free nilpotent or discriminated by filpotent p-groups of finite exponent. 